\title{
Covert Attention as a Paradigm for Subject-Independent Brain-Computer Interfacing
}

\author{
Hans J.P. Wouters ${ }^{1}$, Marcel A.J. van Gerven ${ }^{2,1}$, Matthias S. Treder ${ }^{3}$, \\ Tom Heskes ${ }^{1,2}$, and Ali Bahramisharif ${ }^{1,2}$ \\ 1 Radboud University Nijmegen, Institute for Computing and Information Sciences, \\ Nijmegen, The Netherlands \\ 2 Radboud University Nijmegen, Donders Institute for Brain, Cognition and \\ Behaviour, Nijmegen, The Netherlands \\ 3 Machine Learning Laboratory, Berlin Institute of Technology, Berlin, Germany
}

\section{Introduction}

Covert attention has been introduced as a paradigm for gaze-independent brain computer interfacing (BCI) $[6,7,17,15,2,14]$.As we know that the applicability of a BCI system depends on the consistency of its paradigm over a group of subjects, one important feature of a BCI is its subject-independence. To generalize a BCI to be applicable for new users, one can think of either to use a machine learning algorithm above a standard BCI to improve the generalization or to choose a more appropriate paradigm. Various machine learning algorithms have been applied on imaginary movement based paradigms towards having a subject-independent BCI $[8,16,3,1,10]$, yet there are a few efforts such as [13] on testing other paradigms in this context. In this paper we show that covert attention can be used as a robust paradigm for subject-independent brain computer interfacing.

\section{Methods}

\section{$2.1 \quad$ Data}

Data from the experiment of Treder et al. [14] was used. In the main experiment, eight healthy participants performed a cued visual attention task. First, a white central fixation dot surrounded by six white target discs was presented. A cue appearing for $200 \mathrm{~ms}$ in the center of the screen indicated the target location. Participants had to shift attention to the cued disc while strictly fixating the central dot. After a variable duration (500-2000 ms) the target appeared for 200 $m s$ in the disc as either $a$ ' + ' or a ' $x$ '.

\footnotetext{
* All authors gratefully acknowledge the support of the BrainGain Smart Mix Programme of the Netherlands Ministry of Economic Affairs and the Netherlands Ministry of Education, Culture and Science.
} 
Each participant completed 600 trials in six blocks of 100 trials with twominute breaks between blocks. Cues were valid in $80 \%$ of the trials. In the other $20 \%$ of the cases, the target appeared at a different random location. To ensure that the participants direct their attention immediately after the appearance of the cue, $30 \%$ of the trials featured a short target latency of $500 \mathrm{~ms}$. In the remaining trials, the target latency was randomized between $500 \mathrm{~ms}$ and $2000 \mathrm{~ms}$ in order to ensure that attention is sustained continuously until target appearance. Two vertical and horizontal EOG bipolar channels were used to monitor eye-movements. In this paper we only consider the trials which were $2000 \mathrm{~ms}$ long and we used the same preprocessing steps as the ones taken in [14].

\subsection{Classifiers}

The first algorithm that we used is a correlation based classifier (Cor) which compares the Pearson correlation coefficient of a trial to a class template. The class templates are the average of all trials belonging to a class in the training set. A test sample is classified as the class which corresponds to the template with which the object has the highest correlation $[5,9]$. A small variation of Cor is also used in this paper, which is based on replacing correlation with the Euclidian distance. In this paper, this will be called the closest template classifier (CT). The results of Cor and CT will be compared to Fisher linear discriminant analysis (FLDA).

Evaluation All methods are evaluated in a leave-one-out cross-validation scheme. Accuracies shown are calculated by averaging accuracies of all subjects and all pairs of directions, 120 in total.

To compare different models, e.g. the decisions made by two classifiers 'A' and

'B', Salzberg [12] proposed that $p$-value can be calculated by $p=\sum_{s}^{n} \frac{n !}{s !(n-s) !}(0.5)^{n}$ where $n$ is the number of examples that classifier ' $\mathrm{A}$ ' differs from classifier ' $\mathrm{B}$ ', $\mathrm{s}$ is the number of times classifier ' $\mathrm{A}$ ' is right while classifier ' $\mathrm{B}$ ' is wrong, and $p$ is the probability of this result for a one-sided test. Methods are significant when their results are significantly better, at the $1 \%$ confidence level, than a control labeling, where all trials in the problem are assigned the majority class. To check whether two classifiers are significantly different or not, a two-sided test is more appropriate and the $p$-value is to be doubled. To correct for multiple comparisons, obtained $p$-values are Bonferroni corrected for both the number of pairs of conditions and the number of subjects.

\subsection{Subject-independent learning}

For subject-independent learning the goal is to decode the direction of attention of a subject without any previous class information about that particular subject. We use two different strategies for subject-independent learning: pooling and voting. In the first strategy, all trials except the ones belonging to the test subject are pooled in a single training set and used to build a classifier. This 
model is then used to classify data of the test subject. In the second strategy, we do not pool trials of all subjects together to generate classifiers' model, but we train one model per subject, and let each model get a vote in predicting the class of the unseen trials. Then the winning class is the one that gets more votes. Evaluation was done using a leave-one-subject-out (LOSO) strategy. Pooling and voting are shown with postscripts ' $\mathrm{P}$ ' and ' $\mathrm{V}$ ' in the rest of this paper.

\section{$3 \quad$ Results}

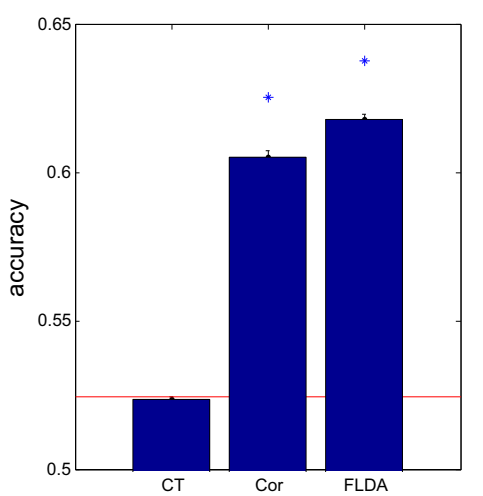

(a)

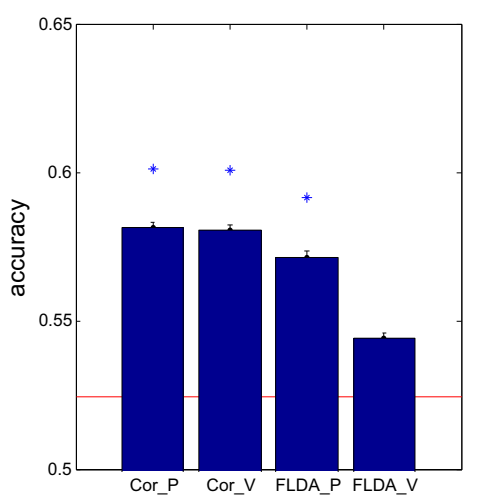

(b)

Fig. 1. (a) Average accuracies of different methods. Stars indicate the method is significantly better than the baseline (majority class). Bars indicate the standard deviation of the mean accuracy. Cor and FLDA are not significantly different from each other. Baseline performance is shown with a straight line. (b) Average accuracies of different methods for subject-independent learning. The postscript ' $\mathrm{V}$ ' and ' $\mathrm{P}$ ' stand for voting and pooling, respectively. Star indicates the method is significantly better than the baseline (majority class). Bars show the standard deviation of the mean accuracy. All methods except from the FLDA with voting are not significantly different from each other. Baseline performance is shown with a straight line.

Figure 1a shows the average classification performances over all pairs of directions and all subjects. Cor and FLDA perform significantly better than the majority class labeling, while CT does not. After observing the low performance of CT, we dropped it for further analysis. The results of subject-independent learning are shown in Fig. 1b. All methods except FLDA_V perform significantly better than the majority class labeling.

Classification performance of Cor_P and FLDA_P versus Cor and FLDA is shown in Fig. 2. There is a correlation of $0.78(p<0.001)$ between the two sets of performances for Cor and $0.52(p<0.001)$ for FLDA. This result implies that the performance of our BCI system highly depends on the subjects and how they 


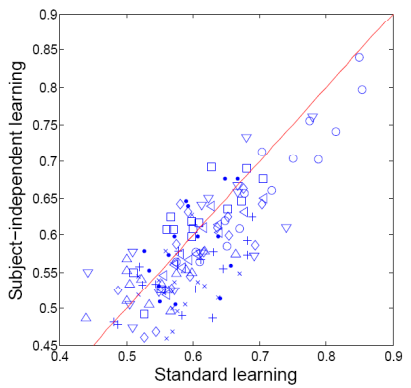

(a)

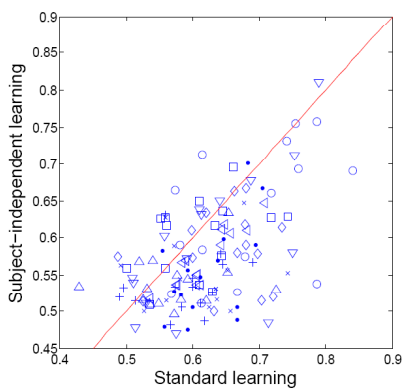

(b)

Fig. 2. Classification performance of Cor_P (a) and FLDA_P (b) versus Cor and FLDA, respectively. Different symbols stand for different subjects. For all subjects, all pairs of directions are included.

perform the task and not whether the BCI system is trained using data of the same subject or not.

\section{Discussion}

We first replicated the classification results reported in [14] using different algorithms. We showed that the pattern correlation classifier (Cor) and linear discriminant analysis (FLDA) give significant classification results for our covert attention BCI paradigm. Average accuray of FLDA shown in Fig. 1a is the same as the results reported in [14]. The variation to the pattern correlation classifier, called the closest template classifier $(\mathrm{CT})$, did not give significant results. This implies that the relative differences of the features is more important than the Euclidean distance between the features and the templates, as the relative differences are also picked up with correlation.

Significant results are obtained for subject-independent learning. Figure $1 \mathrm{~b}$ shows that FLDA_P performs significantly better than FLDA_V, whereas there is no difference between the rest. It means that linear hyper-planes made out of FLDA_V that separate conditions of each subject are very subjective and cannot be generalized.

The high correlation between the two performances shown in Fig. 2 suggests that although the overall performance of subject-independent learning is lower than the standard approach, almost the same pairs of directions can be classified significantly in a leave-one-subject-out setting. Furthermore, Fig. 2a shows that we can get a high performance of 0.84 for a well-performing subject, even if we do not train the classifier with his own data.

We expect to improve the classification performances by utilizing more appropriate techniques such as canonical correlation analysis [11] or hierarchical Bayesian framework [4], which remains to be tested in future works. 


\section{References}

1. M. Alamgir, M. Grosse-Wentrup, and Y. Altun. Multitask learning for braincomputer interfaces. Journal of Machine Learning Research - Proceedings Track, pages $17-24,2010$.

2. A. Bahramisharif, M.A.J. van Gerven, T. Heskes, and O. Jensen. Covert attention allows for continuous control of brain-computer interfaces. European Journal of Neuroscience, 31(8):1501-1508, 2010.

3. S. Fazli, F. Popescu, M. Danóczy, B. Blankertz, K.R. Müller, and C. Grozea. Subject-independent mental state classification in single trials. Neural networks, 22(9):1305-1312, 2009.

4. A. Gelman, J.B. Carlin, H.S. Stern, and D.B. Rubin. Bayesian data analysis. CRC press, 2004.

5. J.V. Haxby, M.I. Gobbini, M.L. Furey, A. Ishai, J.L. Schouten, and P. Pietrini. Distributed and overlapping representations of faces and objects in ventral temporal cortex. Science, 293(5539):2425, 2001.

6. S.P. Kelly, E.C. Lalor, C. Finucane, G. McDarby, and R.B. Reilly. Visual spatial attention control in an independent brain-computer interface. IEEE T. Biomed. Eng., 52(9):1588-1596, 2005.

7. S.P. Kelly, E.C. Lalor, R.B. Reilly, and J.J. Foxe. Visual spatial attention tracking using high-density SSVEP data for independent brain-computer communication. IEEE T. Neur. Sys. Reh., 13(2):172-178, 2005.

8. M. Krauledat, M. Tangermann, B. Blankertz, and K.R. Müller. Towards zero training for brain-computer interfacing. PLoS One, 3(8):e2967, 2008.

9. M. Misaki, Y. Kim, P.A. Bandettini, and N. Kriegeskorte. Comparison of multivariate classifiers and response normalizations for pattern-information fMRI. Neuroimage, 53(1):103-118, 2010.

10. B. Reuderink, J. Farquhar, M. Poel, and A. Nijholt. A subject-independent braincomputer interface based on smoothed, second-order baselining. In Annual International Conference of the IEEE Engineering in Medicine and Biology Society $(E M B C)$, page to appear, 2011.

11. I. Rustandi, M.A. Just, and T.M. Mitchell. Integrating multiple-study multiplesubject fMRI datasets using canonical correlation analysis. In Proceedings of the MICCAI 2009 Workshop. Citeseer, 2009.

12. S.L. Salzberg. On comparing classifiers: Pitfalls to avoid and a recommended approach. Data Mining and knowledge discovery, 1(3):317-328, 1997.

13. R.S. Schaefer, R.J. Vlek, and P. Desain. Decomposing rhythm processing: electroencephalography of perceived and self-imposed rhythmic patterns. Psychological research, pages 1-12, 2010.

14. M.S. Treder, A. Bahramisharif, N.M. Schmidt, M.A.J. van Gerven, and B. Blankertz. Brain-computer interfacing using modulations of alpha activity induced by covert shifts of attention. Journal of neuroengineering and rehabilitation, $8(1): 24,2011$.

15. M.A.J. van Gerven, A. Bahramisharif, T. Heskes, and O. Jensen. Selecting features for BCI control based on a covert spatial attention paradigm. Neural Networks, 22(9):1271-1277, 2009.

16. M.A.J. van Gerven, C. Hesse, O. Jensen, and T. Heskes. Interpreting single trial data using groupwise regularisation. NeuroImage, 46(3):665-676, 2009.

17. M.A.J. van Gerven and O. Jensen. Attention modulations of posterior alpha as a control signal for two-dimensional brain-computer interfaces. Journal of neuroscience methods, 179(1):78-84, 2009. 